\title{
Expression of the SARS-CoV-2 cell receptor gene ACE2 in a wide variety of human tissues
}

\author{
Meng-Yuan $\mathrm{Li}^{1,2}$, Lin Li ${ }^{1,2}$, Yue Zhang ${ }^{3,4,5}$ and Xiao-Sheng Wang ${ }^{1,2^{*}}$
}

\begin{abstract}
Background: Since its discovery in December 2019, severe acute respiratory syndrome coronavirus 2 (SARS-CoV-2) has infected more than 2180000 people worldwide and has caused more than 150000 deaths as of April 16, 2020. SARS-CoV-2, which is the virus causing coronavirus disease 2019 (COVID-19), uses the angiotensin-converting enzyme 2 (ACE2) as a cell receptor to invade human cells. Thus, ACE2 is the key to understanding the mechanism of SARS-CoV-2 infection. This study is to investigate the ACE2 expression in various human tissues in order to provide insights into the mechanism of SARS-CoV-2 infection.

Methods: We compared ACE2 expression levels across 31 normal human tissues between males and females and between younger (ages $\leq 49$ years) and older (ages $>49$ years) persons using two-sided Student's $t$ test. We also investigated the correlations between ACE2 expression and immune signatures in various tissues using Pearson's correlation test.
\end{abstract}

Results: ACE2 expression levels were the highest in the small intestine, testis, kidneys, heart, thyroid, and adipose tissue, and were the lowest in the blood, spleen, bone marrow, brain, blood vessels, and muscle. ACE2 showed medium expression levels in the lungs, colon, liver, bladder, and adrenal gland. ACE2 was not differentially expressed between males and females or between younger and older persons in any tissue. In the skin, digestive system, brain, and blood vessels, ACE2 expression levels were positively associated with immune signatures in both males and females. In the thyroid and lungs, ACE2 expression levels were positively and negatively associated with immune signatures in males and females, respectively, and in the lungs they had a positive and a negative correlation in the older and younger groups, respectively.

Conclusions: Our data indicate that SARS-CoV-2 may infect other tissues aside from the lungs and infect persons with different sexes, ages, and races equally. The different host immune responses to SARS-CoV-2 infection may partially explain why males and females, young and old persons infected with this virus have markedly distinct disease severity. This study provides new insights into the role of ACE2 in the SARS-CoV-2 pandemic.

Keywords: SARS-CoV-2, COVID-19, SARS-CoV-2 cell receptor, Angiotensin-converting enzyme 2, Gene expression, SARS-CoV-2 pandemic, Immune signatures

\footnotetext{
* Correspondence: xiaosheng.wang@cpu.edu.cn

'Biomedical Informatics Research Lab, School of Basic Medicine and Clinical

Pharmacy, China Pharmaceutical University, Nanjing 211198, China

${ }^{2}$ Big Data Research Institute, China Pharmaceutical University, Nanjing

211198, China

Full list of author information is available at the end of the article
}

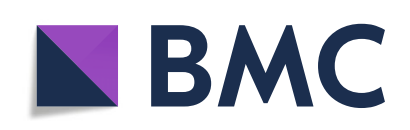

(- The Author(s). 2020 Open Access This article is licensed under a Creative Commons Attribution 4.0 International License, which permits use, sharing, adaptation, distribution and reproduction in any medium or format, as long as you give appropriate credit to the original author(s) and the source, provide a link to the Creative Commons licence, and indicate if changes were made. The images or other third party material in this article are included in the article's Creative Commons licence, unless indicated otherwise in a credit line to the material. If material is not included in the article's Creative Commons licence and your intended use is not permitted by statutory regulation or exceeds the permitted use, you will need to obtain permission directly from the copyright holder. To view a copy of this licence, visit http://creativecommons.org/licenses/by/4.0/. The Creative Commons Public Domain Dedication waiver (http://creativecommons.org/publicdomain/zero/1.0/) applies to the data made available in this article, unless otherwise stated in a credit line to the data. 


\section{Background}

The outbreak of severe acute respiratory syndrome coronavirus 2 (SARS-CoV-2) is rapidly spreading across the world and have caused a global health emergency [1]. Patients infected with SARS-CoV-2 have mainly displayed pneumonia-associated symptoms, including fever, cough, shortness of breath, sputum production, and myalgia or fatigue [2, 3], indicating that SARSCoV-2 primarily infects the respiratory tract and causes acute respiratory disease. However, SARS-CoV-2 infection may result in symptoms of diseases associated with other tissues, such as digestive (diarrhea, poor appetite, nausea, and vomiting), nervous (confusion and headache), and cardiovascular (palmus, chest distress, and cardiac injury) systems [2, 3]. In addition, some studies have indicated that person-to-person transmission of SARS-CoV-2 can occur by routes outside of the respiratory tract [4]. A study of 99 patients infected with SARS-CoV-2 showed that females were less susceptible to infection than males, and older males with comorbidities were more likely to be infected with SARSCoV-2 [3]. Like SARS-related coronavirus (SARS-CoV) [5], SARS-CoV-2 uses the angiotensin-converting enzyme 2 (ACE2) as a host cell receptor [6-8]. A recent study uncovered that $A C E 2$-expressing lung cells were more abundant in Asian males [9], potentially explaining the elevated susceptibility of males to SARS-CoV-2 infection. Nevertheless, the findings from that study are not sufficiently convincing due to a small number of samples being analyzed.

In this study, we analyzed the expression of $A C E 2$ in various normal human tissues using the datasets from the Genotype-Tissue Expression (GTEx) project [10] and The Cancer Genome Atlas (TCGA) program (https://portal.gdc.cancer.gov/). We compared ACE2 expression levels across 31 human tissues, between males and females, and between younger and older persons in these individual tissues. Furthermore, we analyzed the correlations between ACE2 expression levels and immune signature enrichment levels in individual tissues.

\section{Methods}

\section{Datasets}

We downloaded the GTEx RNA-Seq gene expression profiling datasets (RSEM normalized) for 31 human normal tissues from the UCSC Xena project (https:// xenabrowser.net/datapages/). We downloaded the TCGA RNA-Seq gene expression profiling datasets (RSEM normalized) for 12 human normal tissues from the Genomic Data Commons Data Portal (https://portal.gdc.cancer.gov/). All gene expression values were added to 0.001 and then log2-transformed before analysis.
Evaluation of the immune signature enrichment levels in tissue

We defined the enrichment level of an immune signature in tissue as the mean expression level of marker genes of the immune signature in the tissue. We analyzed four immune signatures, including CD8+ T cells, interferon response, B cells, and natural killer (NK) cells. The CD8+ T cell marker genes included CD2, CD247, CD28, CD3D, CD3E, CD3G, CD8A, ICAM1, ITGAL, ITGB2, PTPRC, and THY1 [11]. The interferon response marker genes included IFIT1, IFIT2, IFIT3, IRF7, ISG20, $M X 1, M X 2, R S A D 2, T N F S F 10$, GPR146, SELP, and AHR [12]. The B cell marker genes included $B A C H 2, B A N K 1$, BLK, BTLA, CD79A, CD79B, FCRL1, FCRL3, HVCN1, and RALGPS2 [12]. The NK cell marker genes included KLRC1 and KLRF1 [12].

\section{Statistical analysis}

We used Pearson's correlation test to calculate the correlations between ACE2 expression levels and immune signature enrichment levels in individual tissues. We used a Student's $t$ test (two-sided) to compare ACE2 expression levels between males and females, between younger (ages $\leq 49$ years) and older (ages $>49$ years) persons, and between Asian and non-Asian races in individual tissues. The adjusted $P$ value estimated by the Benjamini and Hochberg method [13] was used to adjust for multiple tests.

\section{Results}

\section{ACE2 expression in various human tissues}

Among the 31 GTEx human tissues, the small intestine, testis, kidneys, heart, thyroid, and adipose tissue had the highest ACE2 expression levels, while blood, spleen, bone marrow, brain, blood vessels, and muscle had the lowest ACE2 expression levels (Fig. 1a). In the lungs, colon, liver, bladder, and adrenal gland, ACE2 showed medium expression levels (Fig. 1a). These results suggest that $A C E 2$ is expressed in a wide variety of human tissues in addition to the lungs.

Furthermore, the Human Protein Atlas (HPA) database (http://www.proteinatlas.org/) showed that the ACE2 protein had relatively high expression levels in the duodenum, small intestine, gallbladder, kidneys, testis, seminal vesicle, colon, rectum, and adrenal gland. The HPA database also showed that the gastrointestinal tract (duodenum, small intestine, colon, and rectum), kidney, gallbladder, and male tissues (testis and seminal vesicle) had high expression levels of both ACE2 gene and protein.

Taken together, these data indicate that: i) SARS-CoV2 may infect other human tissues in addition to lungs, and ii) males may be more susceptible to SARS-CoV-2 infection than females.

We further compared ACE2 expression levels between males and females in 22 individual human tissues using 

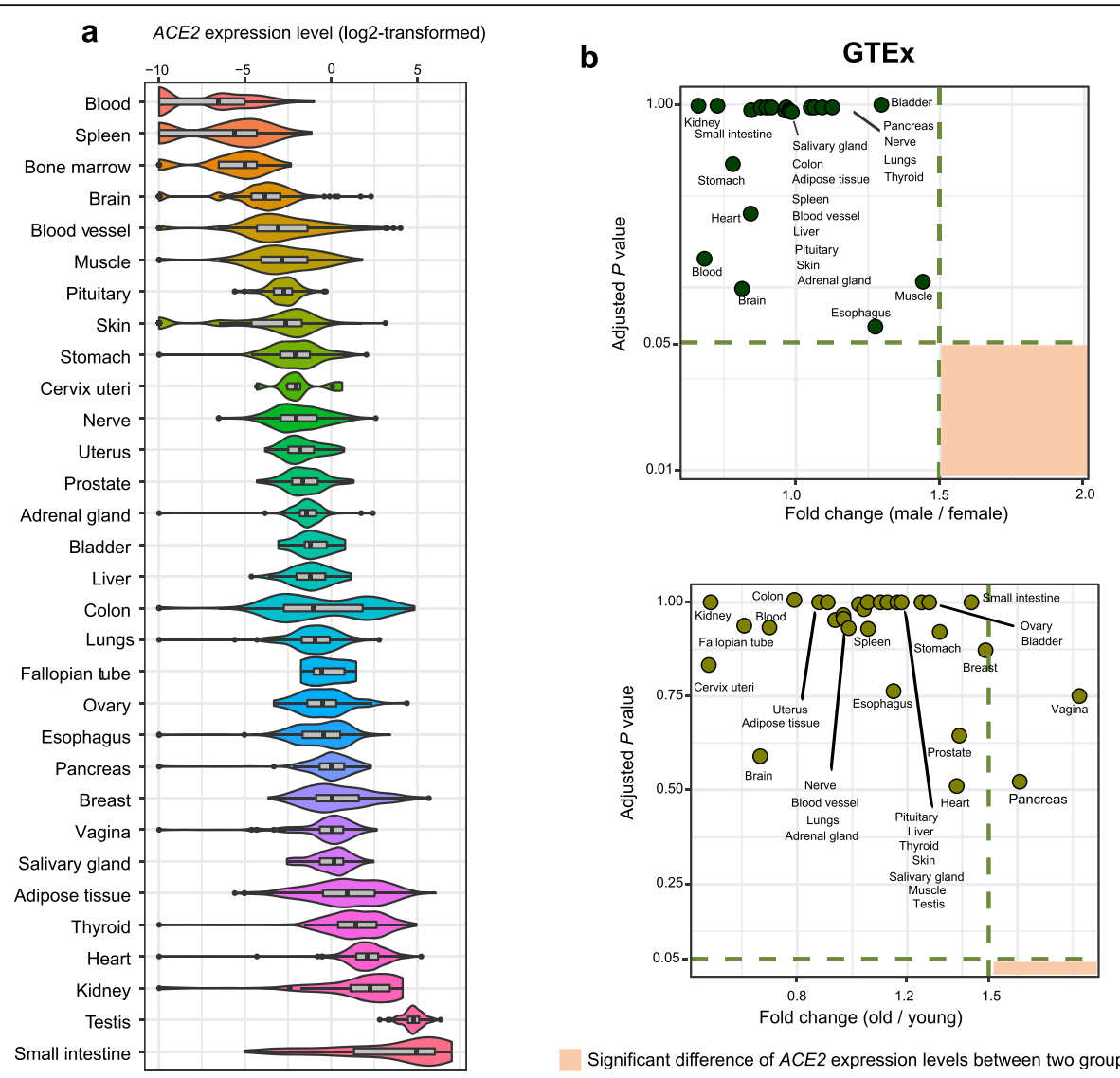

Significant difference of ACE2 expression levels between two groups
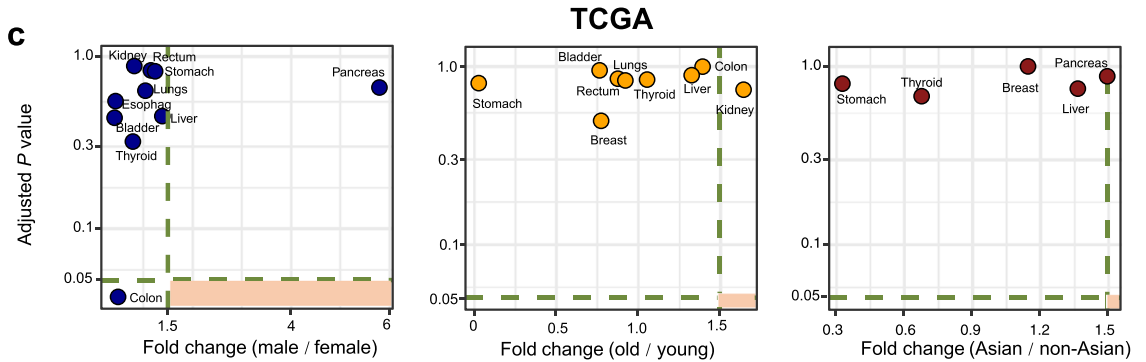

Fig. 1 ACE2 expression in various human tissues. a Comparison of ACE2 expression levels across 31 human tissues in GTEx [10]. Comparison of ACE2 expression levels between males and females and between younger (ages $\leq 49$ years) and older (ages > 49 years) groups in individual human tissues in GTEx (b) and TCGA (c). In TCGA, a comparison of ACE2 expression levels between Asian and non-Asian races in five normal human tissues was also performed (c). Two-sided Student's $t$ test was used in $(\mathbf{b}, \mathbf{c})$. The adjusted $P$ value was calculated by the Benjamini and Hochberg method [13]. ACE2: Angiotensin-converting enzyme 2; GTEx: Genotype-Tissue Expression; TCGA: The Cancer Genome Atlas

the GTEx datasets and found no statistically significant difference between males and females in any tissue using a threshold of adjusted $P$ value $<0.05$ and fold change $>$ 1.5 (Fig. 1b). We also compared ACE2 expression levels between younger (ages $\leq 49$ years) and older (ages $>49$ years) populations and did not observe a statistically significant difference between the groups in any tissue with a threshold of adjusted $P$ value $<0.05$ and fold change $>1.5$ (Fig. 1b). Likewise, in TCGA datasets, ACE2 was not differentially expressed between males and females or between younger and older groups in any tissue (Fig. 1c), consistent with the results in GTEx. In TCGA, we also compared ACE2 expression levels between Asian and non-Asian races in five normal tissues (stomach, thyroid, breast, liver, and pancreas), and did not find a significant difference between them in any tissue (Fig. 1c).

\section{Association of ACE2 expression with immune signatures} We analyzed the correlations between $A C E 2$ expression levels and immune signature enrichment levels (CD8+ T 
cells, interferon response, B cells, and NK cells) in various male and female human tissues. In the skin, digestive system (esophagus, stomach, colon, and pancreas), brain, and blood vessels, we observed significant positive correlations between ACE2 expression levels and CD8+ $\mathrm{T}$ cell enrichment levels in both males and females
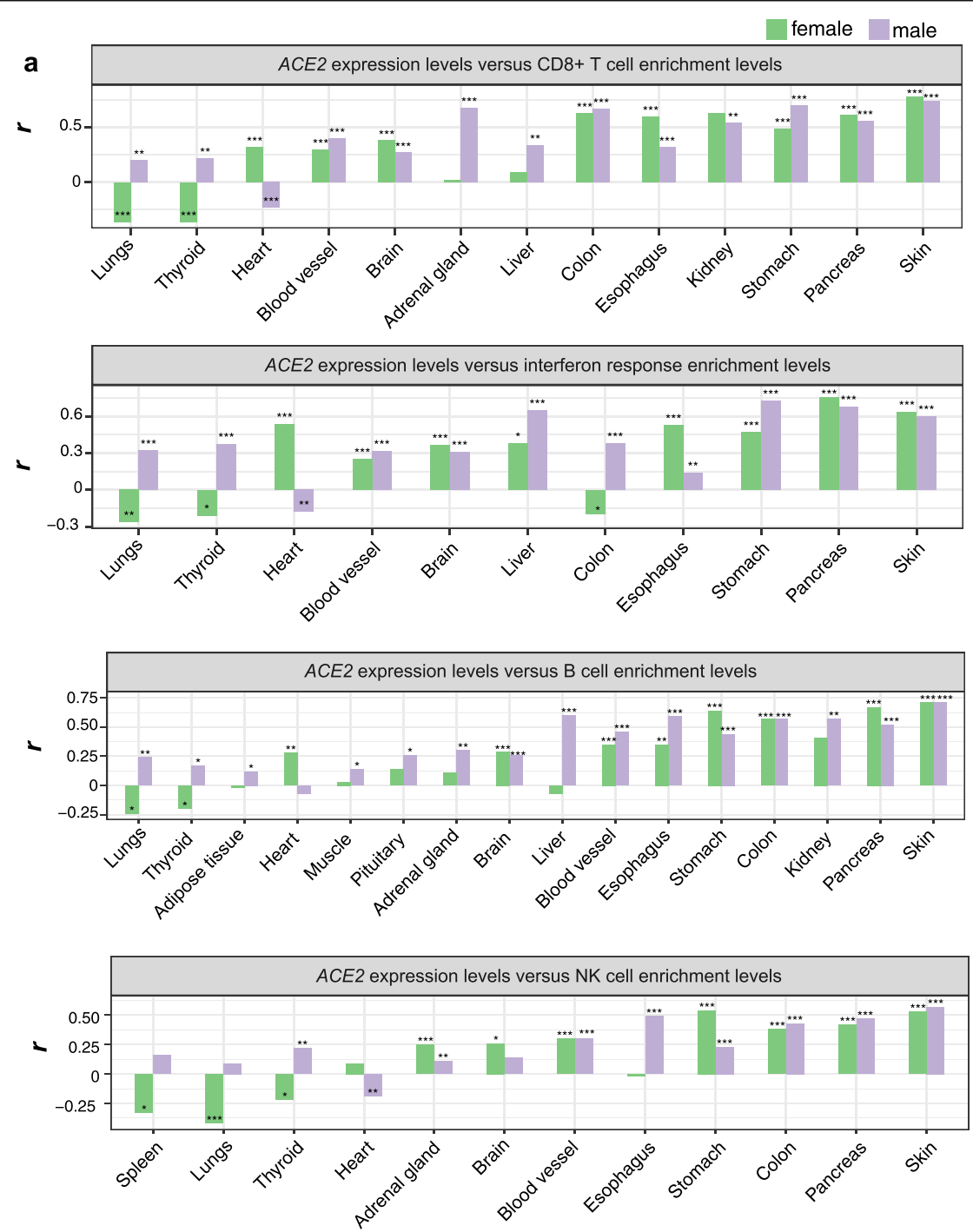

younger group older group

b

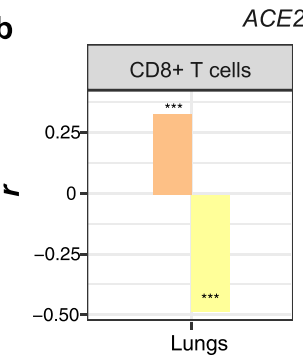

ACE2 expression levels versus immune signature enrichment levels
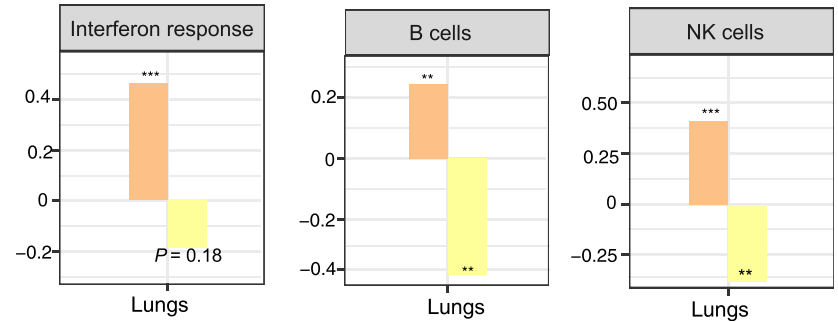

Fig. 2 Association of ACE2 expression with immune signatures in various human tissues. a Correlation between ACE2 expression levels and immune signature enrichment levels in various human tissues in males and females. $\mathbf{b}$ Correlation between ACE2 expression levels and immune signature enrichment levels in the lungs and thyroid of older (ages $>49$ years) and younger (ages $\leq 49$ years) populations. Pearson's correlation test was used to calculate the correlation coefficient $(r)$ and $P$ value in $(\mathbf{a}, \mathbf{b})$. ${ }^{*} P<0.05$, ${ }^{*} P<0.01$, and ${ }^{* * *} P<0.001$. ACE2: Angiotensinconverting enzyme 2; NK: Natural killer 
(Pearson's correlation test, adjusted $P<0.05,0.27 \leq r \leq$ 0.78) (Fig. 2a). In addition, in the thyroid, lungs, adrenal gland, liver, and kidneys, ACE2 expression levels showed significant positive correlations with CD8+ T cell enrichment levels solely in males $(0.20<r<0.68)$. However, in the thyroid and lungs, ACE2 expression levels were negatively correlated with CD8 $+\mathrm{T}$ cell enrichment levels in females $(r=-0.36)$. In the heart, ACE2 expression levels had a negative and a positive correlation with CD8 $+\mathrm{T}$ cell enrichment levels in males $(r=-0.23)$ and females $(r=0.32)$, respectively. Likewise, the interferon response signature had significant positive correlations with $A C E 2$ expression levels in the skin, digestive system (esophagus, stomach, liver, and pancreas), brain, and blood vessels in both males and females $(0.14 \leq r \leq 0.75)$ (Fig. 2a). In the thyroid, lungs, kidneys, adrenal gland, colon, and bladder, ACE2 expression levels had significant positive correlations with the interferon response signature solely in males $(0.32 \leq r \leq 0.82)$. In contrast, in the thyroid, lungs, and colon, ACE2 expression levels were negatively correlated with the interferon response signature in females $(-0.26<r<-0.20)$. In the heart, ACE2 expression levels had a negative and a positive correlation with the interferon response signature in males $(r=-0.18)$ and females $(r=0.54)$, respectively. Similar results were observed for B cells and NK cells (Fig. 2a). Collectively, these results demonstrate the commonality and distinction in the association of $A C E 2$ expression with immune signatures between males and females.

We further analyzed the correlations between $A C E 2$ expression and immune signatures in younger and older populations. In most individual tissues, the correlations between $A C E 2$ expression levels and immune signature enrichment levels displayed consistent trends between younger and older populations. However, in the lungs they had a positive and a negative correlation in the older and younger groups, respectively (Fig. 2b). Again, these results suggest the potential difference in the host immune response to coronavirus infection between young and old persons.

\section{Discussion}

We analyzed the expression of $A C E 2$, the gene encoding the human host cell receptor of SARS-CoV-2 and SARS$\mathrm{CoV}$, in 31 normal human tissues. To the best of our knowledge, this is the first study investigating ACE2 expression across a wide variety of human tissues. We found that although the inflammation of the lungs is the primary symptom of patients with SARS-CoV-2 infection, the lungs had moderate expression of $A C E 2$ among all tissues. This indicates that SARS-CoV-2 may affect other tissues in addition to the lungs, including male tissues (testis and seminal vesicle). This indication has been confirmed by recent publications [2-4]. For example, Holshue et al. uncovered that stool from a patient infected with SARS-CoV-2 was positive for SARS-CoV-2, suggesting that this virus may infect the gastrointestinal tract [5]. Huang et al. reported the virus-related cardiac injury in five patients with SARS-CoV-2 infection [2]. A study of 99 cases of SARS$\mathrm{CoV}-2$-related pneumonia revealed the increased susceptibility of males to infection by this virus [3]. In fact, many cases of SARS-CoV-2 have reported symptoms outside of pneumonia, including diarrhea, nausea, vomiting, confusion, headache, and cardiac injury [2-4]. In addition, the predominant symptoms of patients infected with SARSCoV-2 could be attributed to the fact that the respiratory tract is the readiest transmission approach for the virus.

We found that ACE2 expression levels showed no significant difference between males and females, between younger and older persons, or between Asian and nonAsian races. This indicates that the infection risk of SARS-CoV-2 and SARS-CoV may have no significant association with sex, age, or race. In fact, like SARS-CoV [14], SARS-CoV-2 can affect males and females equally and can infect young and old persons equally [15]. Nevertheless, the mortality risk for SARS-CoV-2 and SARS-CoV infections appears to be associated significantly with sex and age, with a higher risk for males versus females and old versus young populations $[3,14,16]$.

Adaptive and innate immune responses play an important role in fighting off invading coronavirus, even though they may induce a cytokine storm, which is responsible for the immunopathological damage in patients with coronavirus infections [17]. Interestingly, while inflammation of the lungs (pneumonia) is the most common disease caused by coronavirus, the correlations between expression of the ACE2 receptor and immune signatures in the lungs differed between males and females and between young and old persons. This indicates that the host immune response to coronavirus infection could differ between males and females and between young and old persons, partially explaining why males and females and young and old persons have markedly distinct clinical outcomes of coronavirus infections [3, 18]. In addition, it has been demonstrated that lung cells highly expressing ACE2 are more readily infected by SARS-CoV [19]. We found that the correlation between $A C E 2$ expression and immune signatures was negative in the lung tissue of females and younger persons, but it was positive in the lung tissue of males and older persons. Collectively, this means that: i) if the lungs highly expressing ACE2 are infected by SARSCoV-2 in females or young persons, they will have weaker immune signatures, and ii) if the lungs highly expressing ACE2 are infected by SARS-CoV-2 in males or older persons, they will have stronger immune 
signatures. This suggests that an excessive immune response (cytokine storm or immunopathological damage) could be more likely to occur in males and old persons with SARS-CoV or SARS-CoV-2 infection.

This work provides some basis for our next-step investigation of ACE2 expression at protein level. To understand the potential mechanism of interaction between SARS-CoV2 and ACE2, we will explore the possible enrichment and viability of SARS-CoV2 in different tissues alongside different expression level of ACE2. We will try to clarify the survival, death, transfer, and transformation of SARS-CoV-2 in different tissue niches, and explore the virus in vivo inactivation in human body during and after epidemic. In addition, our pilot data suggest that cigarette smoke or nicotine inhalation inhibits the expression of ACE2/AT2R in multiple tissues, including the brain, heart and lungs, thus disrupting the balance within the renin-angiotensin system.

Our study has several limitations. First, the findings from the bioinformatics analysis need to be further validated by experimental and clinical data. In particular, because mRNA expression pattern of ACE-2 is not necessarily the same as its protein expression pattern across tissues due to some factors probably affecting the mapping from mRNA level to protein level, such as post-translational modification, the validation of our mRNA-based findings at protein level is important and worth further investigation. Second, the tissue mRNA expression did not capture the mRNA expression in individual cell types that would be important for precisely understanding the virus-host interaction. An analysis of the single-cell RNA-Seq data may address this issue. However, because the single-cell RNA-Seq data across such a wide variety of human tissues are lacking, it is not realistic to perform such an analysis. Third, the comparison of ACE2 expression levels between Asian and non-Asian races in TCGA datasets was performed in a small number of samples, and thus the associated results need to be verified in larger populations. Finally, our data cannot explain the epidemic divergence between SARS-CoV-2 and SARS-CoV in that SARS-CoV-2 is less virulent but more infectious than SARS-CoV. An in-depth analysis of the genomic and protein structures of both coronaviruses would warrant exploring the mechanism underlying the epidemic divergence between SARS-CoV-2 and SARS-CoV.

\section{Conclusions}

ACE2 is expressed in various human tissues in addition to the lungs, indicating that SARS-CoV-2 may infect other tissues aside from the lungs. ACE2 expression levels have no significant differences between males and females, between young and old persons, and between
Asian and non-Asian races in various human tissues, indicating that SARS-CoV-2 may infect persons with different sexes, ages, and races equally. The correlations between $A C E 2$ expression and immune signatures differ between males and females and between young and old persons in the lungs, indicating that the different host immune responses to SARS-CoV-2 infection may partially explaining why males and females and young and old persons infected with this virus have markedly distinct disease severity. This study provides new insights into the role of ACE2 in the SARS-CoV-2 pandemic and insights into understanding the associations of symptoms, sex, age, and race with SARS-CoV-2 infection.

\section{Abbreviations}

SARS-CoV-2: Severe acute respiratory syndrome coronavirus 2;

ACE2: Angiotensin-converting enzyme 2; GTEx: Genotype-tissue expression;

TCGA: The cancer genome atlas; HPA: Human protein atlas; NK: Natural killer

\section{Acknowledgements}

We thank LetPub (www.letpub.com) for its linguistic assistance during the preparation of this manuscript.

\section{Authors' contributions}

$M L$ performed data analyses and edited the manuscript. LL performed data analyses. $Y Z$ performed data analyses and edited the manuscript. XW conceived of the research, designed the methods, and wrote the manuscript. All authors read and approved the final manuscript.

\section{Funding}

This work was supported by the China Pharmaceutical University (grant number 3150120001 to XW).

\section{Availability of data and materials}

The GTEx and TCGA gene expression profiling datasets for human normal tissues were downloaded from the UCSC Xena project (https://xenabrowser. net/datapages/) and the Genomic Data Commons Data Portal (https://portal. gdc.cancer.gov/), respectively.

\section{Ethics approval and consent to participate}

Ethical approval was waived since we used only publicly available data and materials in this study.

\section{Consent for publication}

Not applicable.

\section{Competing interests}

The authors declare that they have no competing interests.

\section{Author details}

${ }^{1}$ Biomedical Informatics Research Lab, School of Basic Medicine and Clinical Pharmacy, China Pharmaceutical University, Nanjing 211198, China. ${ }^{2}$ Big Data Research Institute, China Pharmaceutical University, Nanjing 211198, China. ${ }^{3}$ Pinghu hospital of Shenzhen University, Shenzhen 440307, China. ${ }^{4}$ Futian Hospital for Rheumatic Diseases, Shenzhen 518000, China. ${ }^{5}$ Department of Rheumatology and Immunology, The First Clinical College of Harbin Medical University, Harbin 150001, China.

Received: 18 February 2020 Accepted: 15 April 2020

Published online: 28 April 2020

\section{References}

1. Horton R. Offline: 2019-nCoV outbreak-early lessons. Lancet. 2020; 395(10221):322.

2. Huang C, Wang Y, Li X, Ren L, Zhao J, Hu Y, Zhang L, Fan G, Xu J, Gu X. Clinical features of patients infected with 2019 novel coronavirus in Wuhan. China. Lancet. 2020; https://doi.org/10.1016/S0140-6736(20)30183-5. 
3. Chen N, Zhou M, Dong X, Qu J, Gong F, Han Y, Qiu Y, Wang J, Liu Y, Wei Y. Epidemiological and clinical characteristics of 99 cases of 2019 novel coronavirus pneumonia in Wuhan, China: a descriptive study. Lancet. 2020; https://doi.org/10.1016/S0140-6736(20)30211-7.

4. Holshue ML, DeBolt C, Lindquist S, Lofy KH, Wiesman J, Bruce H, Spitters C, Ericson K, Wilkerson S, Tural A. First case of 2019 novel coronavirus in the United States. N Engl J Med. 2020; https://doi.org/10.1056/NEJMoa2001191.

5. Li W, Moore MJ, Vasilieva N, Sui J, Wong SK, Berne MA, et al. Angiotensinconverting enzyme 2 is a functional receptor for the SARS coronavirus. Nature. 2003;426(6965):450-4.

6. Xu X, Chen P, Wang J, Feng J, Zhou H, Li X, et al. Evolution of the novel coronavirus from the ongoing Wuhan outbreak and modeling of its spike protein for risk of human transmission. Sci China Life Sci. 2020:1-4.

7. Lu R, Zhao X, Li J, Niu P, Yang B, Wu H, et al. Genomic characterisation and epidemiology of 2019 novel coronavirus: implications for virus origins and receptor binding. Lancet. 2020; https://doi.org/10.1016/S01406736(20)30251-8.

8. Zhou P, Yang XL, Wang XG, Hu B, Zhang L, Zhang W, et al. A pneumonia outbreak associated with a new coronavirus of probable bat origin. Nature. 2020:1-4.

9. Zhao $Y$, Zhao Z, Wang $Y$, Zhou Y, Ma Y, Zuo W. Single-cell RNA expression profiling of ACE2, the putative receptor of Wuhan 2019-nCov. BioRxiv. 2020; https://doi.org/10.1101/2020.01.26.919985.

10. Lonsdale J, Thomas J, Salvatore M, Phillips R, Lo E, Shad S, et al. The genotype-tissue expression (GTEx) project. Nat Genet. 2013;45(6):580.

11. Greenough TC, Straubhaar JR, Kamga L, Weiss ER, Brody RM, McManus MM, et al. A gene expression signature that correlates with CD8+ T cell expansion in acute EBV infection. J Immunol. 2015;195(9):4185-97.

12. Rooney MS, Shukla SA, Wu CJ, Getz G, Hacohen N. Molecular and genetic properties of tumors associated with local immune cytolytic activity. Cell. 2015;160(1-2):48-61.

13. Benjamini $Y$, Hochberg $Y$. Controlling the false discovery rate: a practical and powerful approach to multiple testing. J Royal Stat Soc B. 1995:57:289300.

14. Pang X, Zhu Z, Xu F, Guo J, Gong X, Liu D, et al. Evaluation of control measures implemented in the severe acute respiratory syndrome outbreak in Beijing, 2003. Jama. 2003:290(24):3215-21.

15. Epidemiology Working Group for NCIP Epidemic Response. The epidemiological characteristics of an outbreak of 2019 novel coronavirus diseases (COVID-19) in China. Chin J Epidemiol. 2020;41(2):145-51.

16. Jia N, Feng D, Fang LQ, Richardus JH, Han XN, Cao WC, et al. Case fatality of SARS in mainland China and associated risk factors. Tropical Med Int Health. 2009;14:21-7.

17. Li G, Fan Y, Lai Y, Han T, Li Z, Zhou P, et al. Coronavirus infections and immune responses. J Med Virol. 2020; https://doi.org/10.1002/jmv.25685.

18. Karlberg J, Chong D, Lai W. Do men have a higher case fatality rate of severe acute respiratory syndrome than women do? Am J Epidemiol. 2004; 159(3):229-31.

19. Jia HP, Look DC, Shi L, Hickey M, Pewe L, Netland J, et al. ACE2 receptor expression and severe acute respiratory syndrome coronavirus infection depend on differentiation of human airway epithelia. J Virol. 2005;79(23): $14614-21$.

Ready to submit your research? Choose BMC and benefit from:

- fast, convenient online submission

- thorough peer review by experienced researchers in your field

- rapid publication on acceptance

- support for research data, including large and complex data types

- gold Open Access which fosters wider collaboration and increased citations

- maximum visibility for your research: over $100 \mathrm{M}$ website views per year

At $\mathrm{BMC}$, research is always in progress.

Learn more biomedcentral.com/submissions 